\title{
Architecture definition and operation testing of local electricity markets. The EMPOWER project
}

\author{
Eduard Bullich-Massagué*, Mònica Aragüés-Peñalba*, Pol Olivella-Rosell*, \\ Pau Lloret-Gallego*, Josep-Andreu Vidal-Clos* and Andreas Sumper* \\ *Centre d'Innovació Tecnològica en Convertidors Estàtics i Accionaments (CITCEA-UPC), \\ Departament d'Enginyeria Elèctrica, Universitat Politècnica de Catalunya. ETS d'Enginyeria \\ Industrial de Barcelona, Av. Diagonal, 647, Pl. 2. 08028 Barcelona, Spain.
}

\begin{abstract}
This article describes the results of the European Project Empower. This project is about the creation of a trading platform for local energy exchange in local markets. To do so, a system architecture is proposed, based on the Smart Grid Architecture Model (SGAM). The implementation of the developed platform has been tested in the Prosumer Lab environment. The Prosumer Lab is an installation at the UPC that permits to emulate different use cases by the means of power electronics. The tests showed a successful integration of the software and hardware for the project purpose.
\end{abstract}

Index Terms-Electricity markets; Smart Grid; Platform laboratory; Emulation;

\section{INTRODUCTION}

A significant increase in distributed energy resources (DER) in general, and in distributed generation (DG) in particular, is expected in the coming years [1]. Nowadays, typically small generators are aggregated for their participation in the wholesale electricity market without taking into account their location within the distribution network. In a scenario with high penetration of DG, the power quality could be reduced in terms of voltage limit violations and overloaded lines. Two possible strategies can be considered to face this problem: one is to expand the distribution grid, and the other one is to handle technical constraints with active control to manage local resources such as storage systems and demand response (DR). Grid expansion may require large investments in the future due to, for example, redundant transformers [2]. An alternative requires exploring control algorithms to deal with under-sized distribution grids. Moreover, electricity markets are not designed to deal with generators with null generation costs [3].

Furthermore, the European electricity system in 2050 will integrate millions of electric vehicles and storage units willing to provide energy and flexibility that will be capitalized in distribution grids. In order to guarantee this participation in electric power exchanges, the European Commission has published the Clean Energy Package: Clean energy for all European with the proposal on the Internal Market for Electricity for a revised electricity regulation. The term Local energy community is defined in the Article 16 and it is considered an efficient way to manage energy at community level [1]. In addition, the EU has committed to reduce cut $\mathrm{CO} 2$ emissions by at least $40 \%$ and to increase above $27 \%$ the renewable energy over the total energy consumption by 2030 .
In order to increase the hosting capacity of the current distribution networks without new investments, local markets have merged as a solution for compensating local unbalances.

Some studies, such as [4]-[6], use the concept micromarket or local market. According to the European Network of Transmission System Operators for Electricity (ENTSO-E), in a local market area there are no transmission constraints between the market balance areas [7]. Therefore, for the sake of clarity, the present work applies the term local market to a market structure for distributed participants over a feeder of the distribution network.

Our proposal envisages an integrated wholesale market with geographical distributed multiple local markets. A local market (LM) is understood as an electricity trading platform to sell and buy electricity and flexibility in the neighbourhood. The Smart Energy Service Provider (SESP) is the local market facilitator for the energy community and it is an aggregator for wholesale market agents. This implies that the SESP takes actions to increase market interactions, ensuring enough liquidity. In order to operate these markets, local traders need the SESP platform for sharing information, trading for energy and flexibility, and scheduling actions.

\section{THE EMPOWER H2020 PROJECT}

EMPOWER is a project entitled Local Energy Retail Markets For Prosumer Smart Grid Power Services. It belongs to the topic Modernising the European electricity grid: LCE 7 2014: Distribution grid and retail market of the call Competitive Low-Carbon Energy of the HORIZON 2020 work programme 2014-2015.

The project started in January 2015, with special focus on the local energy markets development. On the one hand, innovative business models are being proposed and promoted to take advantage of the flexibility of generation, load and storage units at distribution level [8]. These business models are setting the operational rules of the local markets operation [9]. On the other hand, an ICT platform is being designed to manage this flexibility based on the suggested business models. The ICT platform enables and manages operation of the system, exchanging the signals between the SESP (Smart Energy Service Provider), brain of the system, and the field elements in order to ensure maximum welfare. 


\section{A. The consortium}

The consortium of the project is constituted by Schneider Electric, a reference in the control of electrical energy and industrial automation, Smart Innovation Norway AS, which manages the NCE (Norwegian Centre of Expertise) cluster of companies and institutions from the academia with special focus on the energetic markets, eSmart, which develops IT solutions, the norwegian distribution company Fredrikstad Energi Nett - FEN, the University de St. Gallen UNISG, with large experience in developing business models, the Centro de Investigación Tecnológica en Accionamientos Eléctricos de la Universidad Politécnica de Cataluña, CITCEA-UPC, characterized by its experience in Mechatronics and Enertronics, with special incidence in the fields of power electronics and digital control, Malta Intelligent Energy Management Agency - MIEMA, energetic agency which targets its research in the promotion of energy efficiency and the grid integration of renewable sources and NewEn Projects $\mathrm{GmbH}$ subsidiary of Diersch \& Schröder (DS), an energetic company with divisions on petroleum sector, renewable energy and IT services.

\section{B. The actual status}

The EMPOWER project promotes micro-generation and the participation of prosumers to provide more flexibility in the electric power exchanges. To do so, the needed business models are being shaped, the SESP ICT platform is being designed and its implementation in pilot sites is under development.

1) Business models: Since the project started, the business models for taking advantage of flexibility in electrical markets have been identified and developed, taking into consideration two dimensions: costs of multiplicity and intervention costs. These business models propose 'coupled services', combining resource-centered and service-centered perspectives and will shape the strategies to operate the system.

2) SESP ICT platform: For ensuring the desired operation of local energy markets, the SESP ICT platform is being developed, together with a SCADA. The original conceptual design of the EMPOWER platform includes three cloud entities communicated among each other: the metering cloud, the control cloud, and the market cloud. The proposed IT architecture unifies these three entities in a unified and flexible technical solution based on Microsoft Azure cloud computing big data PaaS solutions. The type of data being stored range from dynamic metering data to static configuration data and customer data. This data is transmitted through two communication channels linking the SCADA, the SESP and the field equipment. On the one hand, Azure Event Hub is used for all messages from SCADA to SESP and, on the other hand, a SCADA web service is used by SESP for sending control signals. The ICT trading platform has been designed and is being implemented. The implementation has been performed in a first stage in a scaled platform, the so-called Prosumer lab, from CITCEA-UPC, described in Section 4. The second stage consists in the implementation in pilot sites.
3) Pilot sites: The before cited business models will be applied, trough the ICT platform, in the pilot sites where EMPOWER will be tested, located in Norderhaugveien and Sandbakken (Norway), in Wolpertshausen (Germany) and in Gozo (Malta). The pilots' specificities are being taken into consideration before applying the business models, ensuring an appropriate adaptation. To do so, the pilot characteristics in terms of elements installed, technologies used, functionalities and regulatory market constraints have been identified and the use cases to be tested have been selected.

\section{ARChiteCtURE}

The aim of this section is to describe the architecture needed for ensuring the relationships between the different market participants, and to specify control, market and communications interactions for the Smart Energy Service Provider (SESP) platform. The architecture is defined based on the SGAM (Smart Grid Architecture Model) methodology. The SGAM framework has been developed by the Joint Working Group on standards for smart grids from CEN/CENELEC/ETSI in response to the standardization mandate $\mathrm{M} / 490$ to support European Smart Grid deployment. Its methodology is intended to present the design of smart grid use cases by a holistic architecture definition of an overall Smart Grid infrastructure.

Using the own SGAM nomenclature, the three axis used in the Smart Grid model contain the different zones (the hierarchical levels of power system management), domains (which cover the complete electrical energy conversion chain) and interoperability layers (Business, Function, Information, Communication and Component layers to represent interrelations between the different elements of the system).

As reflected in Figure 1, the first level of the architecture (Zone a - Process) is the level where field devices are situated. The communication platform system is situated on the second level, which corresponds to zones b, c and d (field, station and operations), and this platform is responsible for receiving the information from the different field devices and transmits this information to the SESP platform. This information will be treated in a third level by different software applications that form part of the SESP platform (control cloud, metering cloud, and local market cloud in zones d Operation, e Enterprise and $f$ Market respectively).

The components needed in the architecture of EMPOWER and their interactions are represented in the component layer (Figure 2). This component layer is used as a baseline to define the other SGAM layers.

The market cloud manages all transactions and workflows necessary to implement a local energy market. It includes energy scheduling, flexibility, settlement, billing and accounting applications. The control cloud is in charge of managing the orders and schedules that the market cloud defines. It is also equipped with prediction and big data analytics in order to monitor and manage the local market operation. The metering cloud is in charge of monitoring specific clients generation and consumption. It also provides Accounting with periodic 


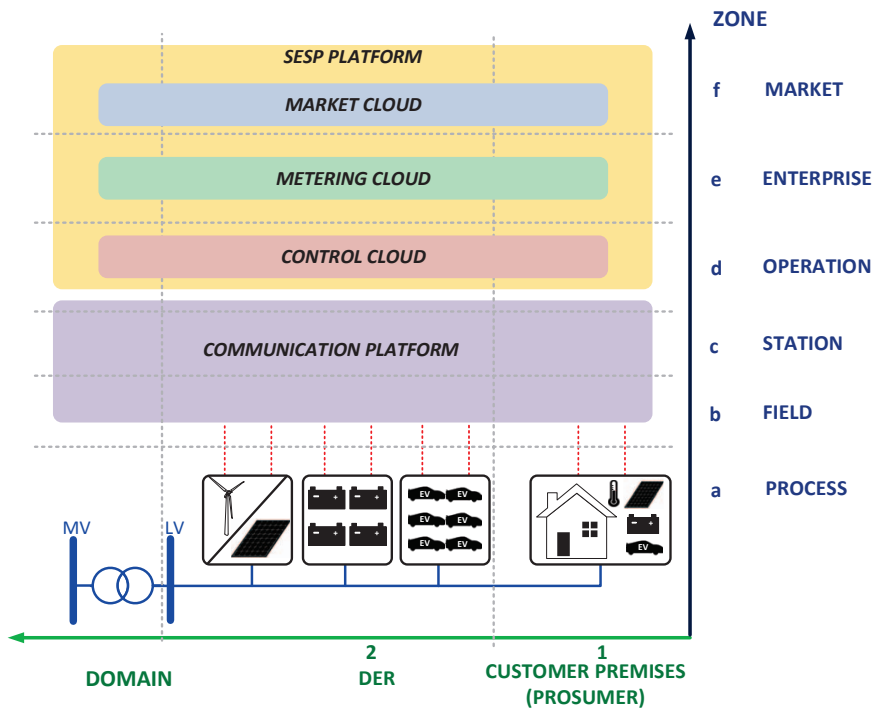

Fig. 1. Empower Communication Architecture based on SGAM

statements on the energy flowing across boundaries within the community and beyond.

The local controller (LC) controls the energy consumption of a load according to a received setpoint in customer premises. The system can consist of several decentralized controllers and a centralized management system to monitor and control the heating, ventilation, air conditioning, light and other facilities within a building. Similarly, DER unit controllers allows the adjustment of its active or reactive power output according to a received setpoint.

For the definition of the SGAM component layer in Figure 2(a), it has been assumed that the DSO and SESP have distinguished roles. In that case, a new metering system is needed in addition to the one used by the DSO. For a hypothetical case where the DSO assumes the SESP functions, the component layer would be the one represented in Figure 2(b), where additionally there is the AMI infrastructure (MDMD, AHE and MDC) to communicate with the smart meters (SM).

The information layer of the SGAM architecture methodology is the layer where the naming and parameters of the data to be exchanged are specified. It details the kind of information to be exchanged between the components of the system (Figure 3). Generation and consumption data from meters are transmitted to the SESP platform through the metering cloud, while generation and consumption setpoints are send from the control cloud to the local controllers at customer premises. However, generation data and setpoints from DER are transmitted through an auxiliary SCADA, which also acts as a gateway to translate from different DER unit controller protocols to the SESP platform.

The communication layer of the SGAM architecture methodology is the layer where the protocol and telecommunication network type are specified. In Figure 3, the generic communication architecture layer for the EMPOWER project concept is described. The main communication standard to be

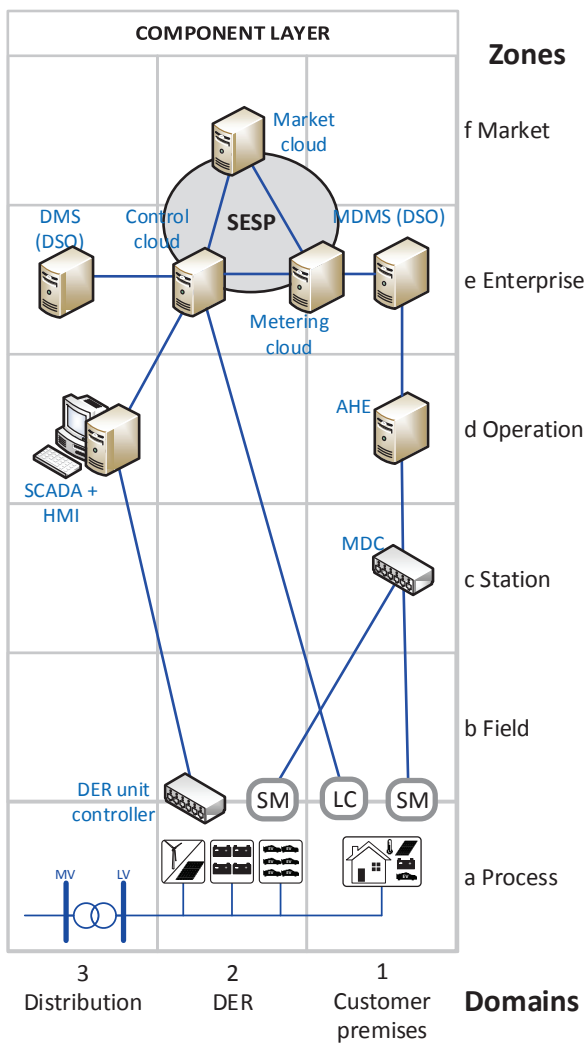

(a) with shared metering information from the DSO

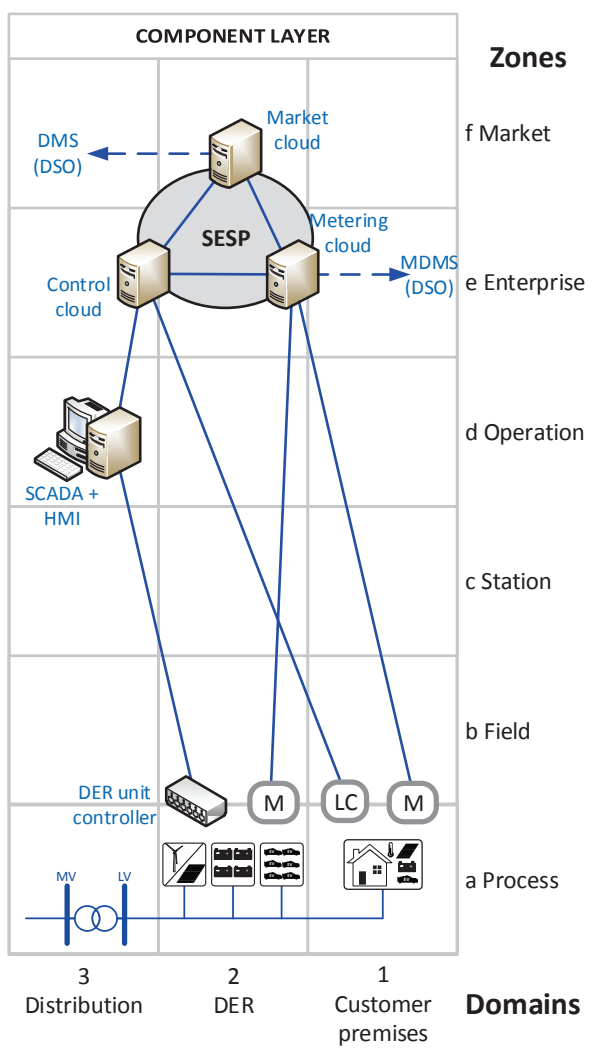

(b) without sharing metering information from the DSO

Fig. 2. Empower SGAM component layers 
applicable, in case that they are needed, for interactions of the SESP platform with external systems such as the EMS or the MDMS is IEC 61968-100, which defines profiles for the communication of CIM messages using Web Services or Java Messaging System. Communications between the SCADA and the DER devices are suggested to be implemented using IEC 60870-5-101 and IEC 60870-5-104. In addition, all the information exchanged between the SCADA system and the control cloud applications will be through Web services. Communication interfaces of the local controllers and the meters are likely to be realised using Web services. These kind of devices are usually connected through a gateway directly to the internet using a subscriber access network. However, these communication interfaces may differ dependent on the chosen components. Alternatively, information for metering purposes can be obtained directly from MDMS through an IEC 61968100 interface.

\section{Prosumer Lab Description}

The proposed architecture has been implemented in a cloud computing system, using Microsoft Azure. In order to test the integration of Hardware and Software, the Prosumer Lab based in the CITCEA-UPC research group has been used.

The Prosumer Lab scheme is shown in Figure 4. It consists of two subsystems. The first one is composed by the emulated systems such as diesel generators, photovoltaic generators, batteries and loads. The emulated devices are then interconnected in the real subsystem through real devices such as power transformers, photovoltaic inverters or battery inverters.

The diesel emulator consists of a rectifier and an inverter. The rectifier serves as a power supply while the inverter is in charge of mimicking the diesel generator behaviour. When the operation is in grid connected mode, this emulator is in charge of supplying active and reactive powers as the real device would do. In grid isolated mode, this emulator imposes the grid voltage and frequency as well as the system inertia. The photovoltaic emulator consists of a rectifier that acts as a current source representing the current that a PV module, a PV string or a PV array would inject under the defined irradiance and temperature conditions. Similarly, the battery emulator consists of a rectifier. But instead of imposing a current, it imposes the dc voltage, which is calculated in function of the state of charge, the charging/discharging power and the battery technology. Finally, the load emulator presents the same architecture as the diesel emulator. But the rectifier imposes the active and reactive power profiles which are previously defined. These profiles can represent a single load or aggregated loads, where each one can be connected or disconnected independently.

The configuration of these emulators is performed through a central microgrid emulator configuration $\mathrm{PC}$, which is interconnected with the emulators through an internal communication network.

On the real subsystem side, the diesel and load emulators are directly connected to the ac 3 phase grid through a power transformer. On the other hand, the PV emulator is

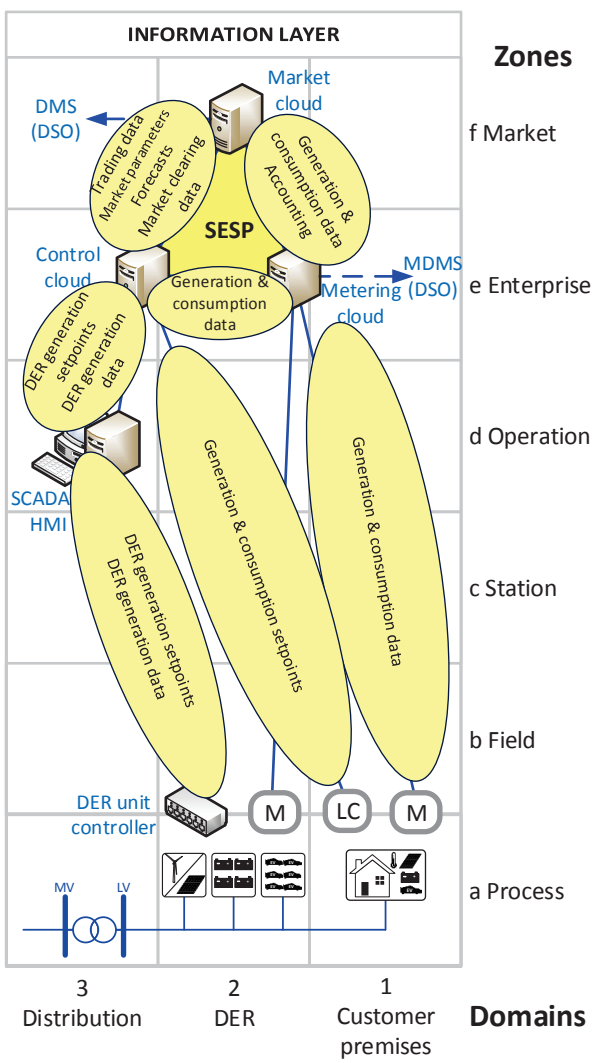

(a) Information layer

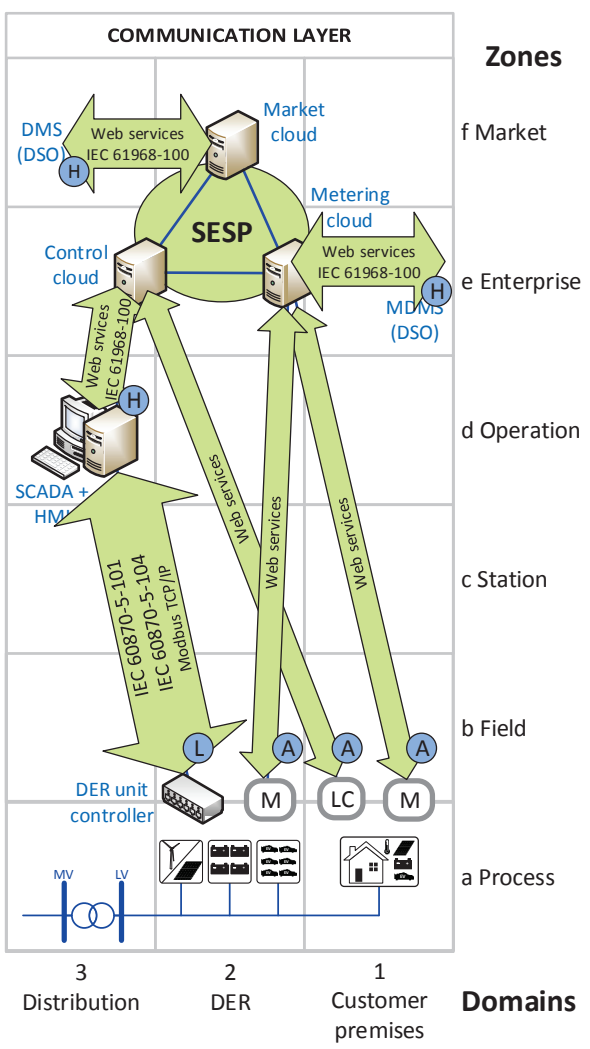

(b) Communication layer

Fig. 3. Information and communication SGAM layers of the EMPOWER system 
interconnected to the ac 3 phase grid through a real dcdc elevator which implements a MPPT algorithm and a real inverter which is in charge to interface the dc to the ac side. The same scheme is shown for the battery interface. In this case, the dc-dc converter controls the charging or discharging active power. The outputs of these interconnected systems (real side) can be connected or disconnected from the main grid thanks to a switch. It permits to perform studies in grid isolated and grid connected modes.

The real devices under test are the SESP, the Supervisory Control And Data Acquisition (SCADA) and the communication infrastructure. The SESP computes its algorithms in the cloud and the output orders are sent to a Supervisory Control And Data Acquisition (SCADA) system through a web service protocol. The SCADA system receives these orders and sends them to the corresponding device (emulator). It is done through a local Ethernet communication network under MODBUS protocol.

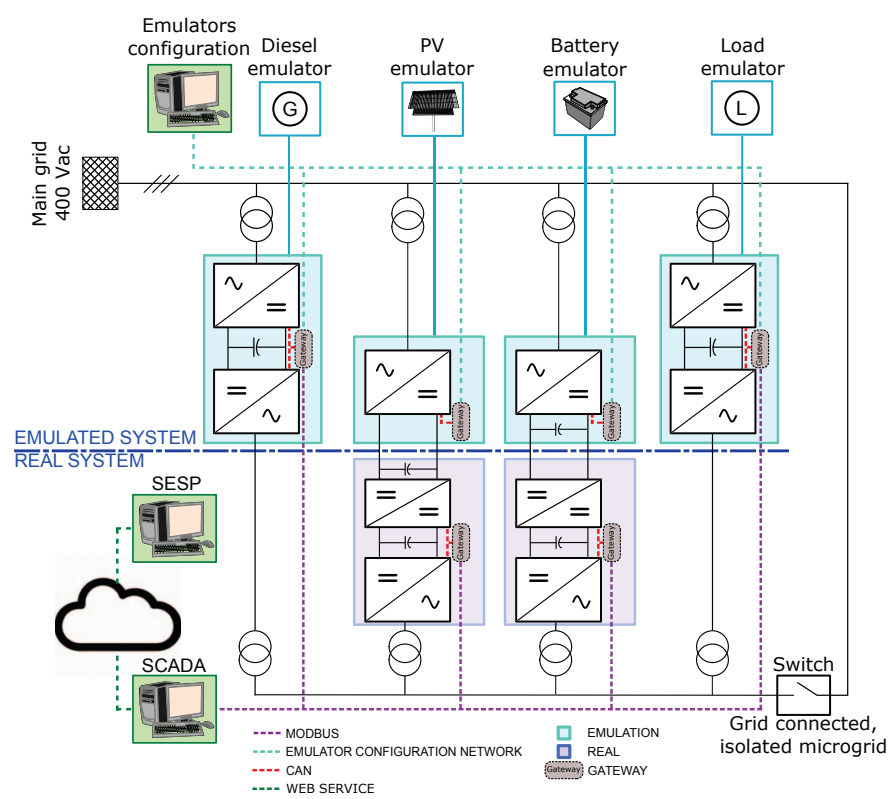

(a) Microgrid emulator scheme

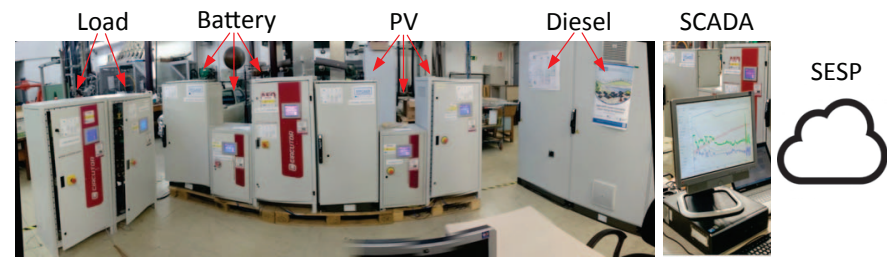

(b) Microgrid photo

Fig. 4. Prosumer Lab description

\section{CONCLUSION}

The need to trade locally electricity has pushed the demand for specialized platforms for such a local trading. The Empower projects shows one solution, using the Smart Energy Service Provider to facilitate such local electricity exchange. The proposed architecture shows a versatile and scalable solution based on cloud services. The integration of the cloud with the utility SCADA works seamless in the case of laboratory tests performed with the Prosumer Lab at the UPC. This Prosumer Lab is a versatile and flexible test bench that can be used to perform test of the designed use cases.

\section{ACKNOWLEDGMENT}

This work has been supported by EMPOWER H2020 project, which has received funding from the European Unions Horizon 2020 research and innovation programme under grant agreement No 646476

\section{REFERENCES}

[1] International Energy Agency (IEA), "Medium-Term Renewable Energy Market Report 2015. Market Analysis and Forecasts to 2020," Tech. Rep., 2015.

[2] R. Poudineh and T. Jamasb, "Distributed generation, storage, demand response and energy efficiency as alternatives to grid capacity enhancement," Energy Policy, vol. 67, pp. 222-231, 2014. [Online]. Available: http://dx.doi.org/10.1016/j.enpol.2013.11.073

[3] M. Ahlstrom, E. Ela, J. Riesz, J. O’Sullivan, B. F. Hobbs, M. O’Malley, M. Milligan, P. Sotkiewicz, and J. Caldwell, "The Evolution of the Market: Designing a Market for High Levels of Variable Generation," pp. 60-66, 2015.

[4] I. Faber, W. Lane, W. Pak, M. Prakel, C. Rocha, and J. V. Farr, "Microenergy markets: The role of a consumer preference pricing strategy on microgrid energy investment," Energy, vol. 74, pp. 567-575, 2014.

[5] A. Kriukov, "Applying a Micro-Market Inside an Electric Vehicles Parking Facility," in Power Engineering Conference (UPEC), 2014 49th International Universities, 2014.

[6] C. W. Lane, W. Pak, M. Prakel, C. Rocha, M. A. J. I. Faber, and J. V. Farr, "Costing Consumer Preferences for a Micro Energy Market," Center for nation reconstruction and capacity development, Tech. Rep. July, 2013.

[7] ENTSO-E, "The harmonised electricity market role model," 2011. [Online]. Available: http://www.ebix.org/documents/role_model_v2011_01.pdf

[8] S. O. Ottesen and A. Tomasgard, "A stochastic model for scheduling energy flexibility in buildings," Energy, vol. 88, pp. 364 - 376, 2015. [Online]. Available: http://www.sciencedirect.com/science/article/pii/S0360544215006301

[9] T. Helms, M. Loock, and R. Bohnsack, "Timing-based business models for flexibility creation in the electric power sector," Energy Policy, vol. 92, pp. 348 - 358, 2016. [Online]. Available: http://www.sciencedirect.com/science/article/pii/S0301421516300763 